due thought. But again, his book provides little account of the relationships between psychopharmacological and purely psychological intervention. Lithium per se may not 'cure' an affective disorder, but there must, one presumes, be guidelines for how it could be used effectively in association with counselling? Some of these issues are broached in the sections on depression, but the level of discussion is low even when the outcome seems sensible enough: "My rule of thumb is that any patient sick enough to require psychotropic medication deserves concomitant psychotherapy and follow-up".

The whole concept of neuropsychiatry receives disappointingly short shrift in a book that aspires to bridge "a longstanding and counterproductive gap between psychology and biology". And nowhere is the shrift shorter than in the paragraphs on electroconvulsive therapy, one of the unfortunate side-effects of which is memory loss. Here Basch writes: "My experience, admittedly not recent, has been that the permanent memory loss in patients receiving electroconvulsive treatment was only for the details of the illness and the treatment itself". This claim may, or may not, be true, but Basch should surely have provided a brief review of the experimental evidence on the topic.

I fear that the prestige of science has outstripped its achievements in relation to our understanding of human beings. There are branches of psychology that truly merit the designation 'scientific', but they are few and far between. Basch's book provides no grounds to include psychotherapy among them. Which is a pity, if for no other reason than that good psychotherapy from a practitioner as competent as Basch could in fact be extremely valuable. But the case might have looked stronger (and the book have been a hundred pages shorter) without the pseudoscience.

John C. Marshall is in the Neuropsychology Unit, part of the Neuroscience Group at the Radcliffe Infirmary, Oxford $O X 26 \mathrm{HE}, U K$.

\section{Cladistic futures}

\section{Philip D. Gingerich}

The Phylogeny and Classification of the Tetrapods. Vol. 1 Amphibians, Reptiles, Birds; Vol. 2 Mammals. Edited by Michael J. Benton. Oxford University Press: 1988. Vol. 1 pp. 377, £50, \$98; Vol. 2 pp. 329, £45, $\$ 90$.

Revolutions in science are like waves crashing on a shore. They arise quietly, gain energy and eventually break thunderously, spraying showers of new perspective that alter our view of the shoreline forever. For the past 20 years, a new wave, cladistics, has been building in systematic biology. It is finally breaking, producing phylogenies and classifications in profusion, and after all the rhetoric we can finally begin to evaluate the results.

The Phylogeny and Classification of the Tetrapods represents part of the proceedings of an international symposium convened in London in 1987 and sponsored by the Systematics Association, the Linnean Society and the Palaeontological Association. Each volume opens with a brief preface by the editor, stating that the intention of the symposium was to try to present balanced coverage of the systematics of all the main tetrapod groups. Reading between the lines, it seems that balance was soon lost in controversy over methods and interpretative details. There are 20 chapters altogether (nine in Vol. 1 , eleven in Vol. 2) written by 34 contributors, virtually all of whom are palaeontologists. Cladistics is now established around the world and readers are mercifully spared the all-too-familiar primer on the inadequacy of the fossil record, parsimony, sister-groups, three-taxon statements and so forth. There are no real surprises, apart from Bishop and Friday's $\alpha$ - and $\beta$-haemoglobin sequences suggesting that birds and mammals should be grouped together.

How has cladistics changed our view of the history of life? The Phylogeny and Classification of the Tetrapods gives us a chance to compare tetrapod phylogeny today with our understanding before cladistics became widely applied. The main differences from pre-cladistic Romer (as seen in his book, Vertebrate Paleontology, published by the University of Chicago Press in 1966), result from a new and sometimes questionable willingness to make precise claims about sequences of branching that occurred over short intervals of geological time (often at or within gaps in the fossil record).

Prizes for data matrices go to Gauthier, Kluge and Rowe (14 amniote taxa $\times 94$ characters, analysed using $P A U P)$, Evans (54 diapsid taxa $\times 226$ characters, analysed by hand), Novacek, Wyss and McKenna (partial matrix of 20 living mammalian orders $\times 67$ characters, analysed in pieces by hand, PAUP or $P H Y S I S)$, Andrews (10 primate taxa $\times 54$ characters, analysed using $P A U P$ ), Prothero, Manning and Fischer (13 ungulate taxa $\times 28$ characters, explored using MacClade), and Gentry and Hooker (39 artiodactyl taxa $\times 116$ characters, analysed using $P A U P$ )

Consistency indices of cladograms based on full matrices range from Gauthier et al.'s 0.71 (29 per cent of state changes incongruent with proposed cladogram), to Andrews's 0.64 (36 per cent incongruence), to Gentry and Hooker's 0.29
(71 per cent incongruence). Bishop and Friday caution that comparing and evaluating all possible tree diagrams is not possible because of limitations on computing time, Gauthier et al. analyse 14 taxa because this is all their mainframe $P A U P$ can branch and bound, and Novacek et al. use software limiting them to full analysis of trees for a maximum of nine taxa. The remaining authors can have little idea whether theirs is the most parsimonious tree.

These volumes reflect the state of cladistics in the late 1980s. The good news is that authors of four out of the 20 chapters (20 per cent) use molecular or morphological information in conjunction with a rigorous algorithm to produce cladograms from data. The first of the bad news is that authors of the remaining chapters $(80$ per cent) produce cladograms by hand in unknown ways, using characters just to diagnose the branches. The next bad news is that consistency indices peak at 71 per cent ( 29 per cent incongruence) - character convergence, parallelism and reversal are common in evolution. But the worst news is that maximum parsimony solutions cannot be computed for problems involving more than 14 or so taxa (these problems are 'NP-complete' in the language of computer science, which means difficulties of combinatorial optimization make them computationally intractable). The index to the first volume lists 800 vertebrate taxa, that to the second volume 800 more. No one will ever compute a maximum parsimony solution showing what we really want to know - the genealogical relationships of these 1,600 taxa (or more). And if we can't do it with a computer then we can't do it by hand.

The problem is that we expect too much of morphology in asking it to tell us the genealogy of organisms as well as what they look like. We ask too much of form that it should tell us time. Fortunately there is an independent record of time in the geological/stratigraphical record. Romer took time from geology rather than morphology, and it looks like he got the main outline of tetrapod evolution right. If each author here and in the future would add one column along the side of his or her matrix of character states giving a geological age for each taxon in the matrix (age is almost always known to good approximation from independent evidence), it would open the possibility of a host of new stratocladistic and stratophenetic approaches to phylogeny that tell us more than character analysis alone ever will, even for large numbers of taxa. Time, after all, is the independent variable in evolution, and time is the dimension biologists expect palaeontologists to bring to our common endeavour.

Philip Gingerich is in the Museum of Paleontology, University of Michigan, Ann Arbor, Michigan 48109-1079, USA. 